\title{
Prediction for Heat Loss in Reheating Furnace due to Partition Wall Installed at Soaking Zone.
}

\author{
Seungjin Lee ${ }^{1}$, Seongkyu See ${ }^{1}$ \\ ${ }^{1}$ POSCO Technical Research Institute, Pohang, Republic of Korea \\ bloodone@posco.com
}

\section{Extended Abstract}

About $25 \%$ of the energy consumption in South Korea's industry is used in the metal industry. It is imperative to reduce energy use in steel mill industry which is a big part of the metal industry. The reheating furnace which is a device that heats the slab to a target temperature (approx. $1100^{\circ} \mathrm{C}$ or higher) using COG (coke oven gas) and BFG (blast furnace gas) is a process that typically uses excessive energy in the rolling process.

The reheating furnace usually consists of pre-heating zone/heating zone/soaking zone and doors for slab charging and discharging. Usually, the heat loss is prevented by closing door during operating, but opening the door is essential when the slab is charged or discharged. When the door is opened, a lot of high temperature gas is escaped out and it is a major cause of heat loss.

A stack is installed at the top of pre-heating zone and the inside of the furnace is constructed with a negative pressure for reducing emission of high temperature gas, but there is no such a device at soaking zone. Also, burner direction at soaking zone is mostly installed directly towards the door, thereby losing a lot of energy at the door.

In this study, it is proposed to install the partition wall on the lower part of a reheating furnace to reduce heat loss at soaking zone. The lower partition wall is constructed with a material that can las for a long time in heat according to the height of the location. It prevents cold outside air inflow during the discharge door open, and reduces high temperature gas directly leaving from the burner to increase energy efficiency.

A 3D unsteady numerical simulation is conducted by Fluent to verifying heat loss at soaking zone. Burner in the upper and lower sections are set as velocity inlet and discharging door side is set as pressure outlet. In addition, the pressure outlet is set for the part connected to heating zone and the negative pressure is set based on the force pulled by the stack installed in pre-heating zone.

The energy efficiency is analyzed by checking the thermocouple installed inside. Also, the volume-average temperature and the flow of fluid is concerned.

The result shows that the installation of the partition wall is effective. If the height of the wall is too low, the intrusion of the outside air cannot be blocked and the high temperature gas form the burner can easily escape. Conversely, if the height is too high, a dead zone occurs and cold air stays in the area, and this cold air lowers the temperature of the lower part of the slab, which may cause the downward movement of the plate during rolling. Optimized height of the wall (wall height/full height) is deduced by simulation from 0.2 to 0.4 